\title{
The influence of underweight and obesity on the diagnosis and treatment of appendicitis in children
}

\author{
Marjolijn E. W. Timmerman ${ }^{1}$ - Henk Groen ${ }^{2} \cdot$ Erik Heineman $^{1}$ • Paul M. A. Broens ${ }^{1,3}$
}

Accepted: 3 June 2016 / Published online: 16 June 2016

(C) The Author(s) 2016. This article is published with open access at Springerlink.com

\begin{abstract}
Purpose The impact of lower body mass index (BMI) on appendicitis has never been addressed. We investigated whether different BMIs affect the diagnosis and treatment of appendicitis in children.

Methods The correlation between BMI and diagnosis accuracy and treatment quality was evaluated by retrospective analysis of 457 children diagnosed with appendicitis. Based on BMI percentiles, patients were classified as either underweight $(n=36)$, normal weight $(n=346)$, overweight $(n=59)$, or obese $(n=16)$. Diagnosis accuracy was measured by negative appendectomy rate, perforation rate, and number of consultations. Treatment quality was measured by complication rate and length of hospital stay.

Results Underweight patients had the highest negative appendectomy (OR 3.00, $P=0.008)$ and complication (OR 2.75, $P=0.041)$ rate. BMI did not influence perforation rate or number of consultations. Both underweight and obese patients stayed in the hospital longer than normal weight patients (regression coefficient $2.34, P=0.001$, and regression coefficient $9.40, P<0.001$, respectively).
\end{abstract}

Marjolijn E. W. Timmerman m.timmerman01@umcg.nl

1 Department of Surgery, Division of Pediatric Surgery, University of Groningen, University Medical Center Groningen, Groningen, the Netherlands

2 Department of Epidemiology, University of Groningen, University Medical Center Groningen, Groningen, the Netherlands

3 Department of Surgery, Anorectal Physiology Laboratory, University of Groningen, University Medical Center Groningen, Groningen, the Netherlands
Furthermore, in obese patients, the hospital stay after open appendectomy was prolonged compared to laparoscopic appendectomy $(P<0.001)$. No such differences were observed in patients with lower BMI.

Conclusions Underweight children are misdiagnosed more often, stay in hospital longer, and experience more postoperative complications than children of normal weight. Obesity is associated with longer hospital stays. Laparoscopic appendectomy might shorten the length of hospital stays in these patients. We conclude that in addition to obesity, underweight should also be considered a risk factor for children with appendicitis.

Keywords Appendectomy $\cdot$ Appendicitis $\cdot$ Body mass index $\cdot$ Obesity $\cdot$ Underweight

\section{Introduction}

Appendectomy is one of the most common surgical interventions and is performed in approximately 1-4 in 1000 children per year [1]. Obesity was reported to negatively affect the time required for and the accuracy of the diagnosis of appendicitis in children [1-3]. Furthermore, obesity was found to affect wound healing due to delay in the recovery of mechanical strength, decreased wound collagen deposition, and an increased risk of inflammation [4]. Therefore, in adult patients, obesity also appears to impact the complication rate and length of hospital stay after appendectomy [5-7]. Nevertheless, reports to the contrary have also been published $[2,8]$.

Whereas obesity is a recognized risk factor for accurate diagnosis and surgical outcomes, the impact of a low BMI has not been addressed. In fact, underweight patients are often excluded from studies evaluating the influence of BMI on outcomes despite the fact that any abnormality, including underweight, may influence a patient's health [9]. It was reported 
that underweight changes physiological processes and may, for instance, lead to malnutrition and osteoporosis [10], impaired functioning of the immune system [11], or disturbed wound healing [12]. Preoperative malnutrition was identified as an important predictor of poor clinical outcomes in adult patients undergoing gastrointestinal operations [13]. Surprisingly, little is known about the correlation between underweight and the diagnosis or surgical outcomes after appendicitis.

Surgical intervention in case of appendicitis involves different procedures, of which the open appendectomy and laparoscopic appendectomy are most frequently performed. The influence of BMI on the outcome of these two types of appendectomy is still under debate. Several studies demonstrated that a laparoscopic appendectomy associates with better outcomes in both obese children and adults in the sense of fewer complications and shorter hospital stays [6, 14, 15], while other studies reported no additional benefit from laparoscopy for obese patients compared to non-obese patients $[16,17]$.

Our first aim was to determine whether BMI, from low (underweight) to high (obesity), influenced the diagnosis of appendicitis in children. Our second aim was to study whether BMI influenced the outcome of the treatment of appendicitis. Our third aim was to analyze which operative technique had a favorable outcome in the different BMI categories.

\section{Materials and methods}

\section{Patients}

We compiled a database of 697 patients (5-18 years old), diagnosed with appendicitis, and who underwent an appendectomy. These patients were referred to the emergency department of University Medical Center Groningen, in the Netherlands, between January 2000 and September 2015.

We excluded 208 patients from the database of 697 patients due to lack of information on weight and/or height (the two parameters required to calculate BMI). Subsequently, we also excluded 32 patients who had a median laparotomy, since this technique is used if complications are expected to occur. Finally, 457 patients were selected for analyses.

The local medical ethics committee approved this study.

\section{Variables}

To study the influence of BMI on the diagnosis of appendicitis, we analyzed the negative appendectomy rate, perforation rate, and number of consultations. The negative appendectomy rate was a measure for the number of patients misdiagnosed with appendicitis according to perioperative findings and/or pathological examination of the removed appendix. The perforation rate was based on the percentage of perforations visible during appendectomy. Consultations were performed by a surgeon in training under supervision of a pediatric or general surgeon. An additional consultation the next day was used for clinical reassessment during which laboratory and/or imaging investigations were repeated. For 55 patients, data were missing regarding the number of consultations required for diagnosing the patient.

To establish a possible correlation between BMI and its influence on the treatment of appendicitis, we investigated the complication rate and length of hospital stay. Data on the length of hospital stay were missing for eight patients. The postoperative complications taken into account were wound infections, abscess, fever lasting for more than 2 days after appendectomy, peritonitis developed after appendectomy, hematoma, bowel obstruction, urinary tract problems, postoperative ileus, readmission, and one or more reoperations.

BMI in children was corrected for age and gender and expressed in percentiles ranging from 0 to 100 , where 0 stood for severe underweight and 100 for extreme obesity [18]. We investigated the following BMI groups: BMI percentile $<5$ (underweight, $n=36$ ), BMI percentile 5-84 (normal weight, $n=346$ ), BMI percentile 85-94 (overweight, $n=59$ ), and BMI $\geq 95$ percentile (obese, $n=16$ ).

To establish possible correlations between BMI and the performed operative techniques, we compared the outcomes after laparoscopic and open appendectomy. Open appendectomy was performed mainly during the first years of this study, while a laparoscopic approach was used more often during the last few years. We, therefore, corrected our analyses for the year in which the appendectomy had been performed. Prophylactic antimicrobial treatment was the same for both operative techniques. Appendectomy was performed within $12 \mathrm{~h}$ of diagnosis, and the operation was not postponed until the next day. Data on the type of appendectomy used were available for 411 patients. All patients underwent standard clinical and laboratory assessment. Information on the use of ultrasound and CT scan was available for 402 patients.

To correct for variables in the multivariable analyses, we used information on the year the appendectomy had been performed and on age, gender, height, weight, operative technique, perforation status, C-reactive protein (CRP) levels, and leukocyte levels of the patients. The variables leukocytosis and increased CRP level were both corrected for age and gender.

\section{Statistical analysis}

We used SPSS 22.0 for Windows (IBM SPSS Inc., Armonk, NY) for the statistical analyses of the data. A descriptive analysis was performed for all the variables. To test if variables were normally distributed, we used the Kolmogorov-Smirnoff and Shapiro-Wilk tests. For normally distributed continuous data, we used the independent-sample Student's $t$ test, and to 
analyze abnormally distributed continuous data, we used the Mann-Whitney $U$ test. We used the chi-square test to compare categorical data. After the univariate analyses, we performed a multivariable analysis to correct for variables such as age and gender to create a better simulation of the actual clinical setting. The multivariable analyses used to determine diagnostic accuracy were corrected for year of appendectomy, age, and gender. Multivariable analyses used to determine treatment quality were corrected for year of appendectomy, age, gender, type of operative technique, perforation status, CRP level, and leukocyte level. We used binary logistic regression to estimate the odds ratio (OR) and $95 \%$ confidence interval $(95 \% \mathrm{CI})$ for the binominal outcomes (negative appendectomy rate, perforation rate, number of consultations, and complication rate). Linear regression was used to estimate the regression coefficient $(B)$ and $95 \%$ CI for the continuous outcome (length of hospital stay). The BMI category "normal weight" was used as a reference value to which all the other BMI categories were compared. We considered $P$ values below 0.05 statistically significant.

To investigate which operative technique had the most favorable outcome in terms of complications and length of hospital stay for the different BMI categories, we used an interaction term. The interaction term indicates whether the influence of one variable depends on the value of another variable, e.g., whether the influence of BMI on the occurrence of complications is different for BMI groups according to surgical technique. If the interaction term is significant $(P<0.05)$, it implies that BMI and the operative technique interact with each other and that therefore, these factors together have a different influence on the outcome of the analyses than separately.

\section{Results}

\section{Characteristics of patients, diagnostic methods, and operative techniques}

The patient characteristics per BMI group are presented in Table 1 . Underweight children were significantly younger than children with normal weight $(P=0.001)$. There was no difference in gender distribution per BMI group.

The clinical and laboratory assessment was the same for each BMI group. Nevertheless, an ultrasound was performed almost twice as often in the underweight group than in the other BMI groups $(P=0.023)$ (Table 1$)$. Age and gender had no significant influence on the use of ultrasound.

In most BMI groups, laparoscopic appendectomy had been performed more often than open appendectomy, except in the case of the underweight group $(P=0.005)$. In these patients, laparoscopic appendectomy was performed equally often as open appendectomy.
Table 1 Patient characteristics, diagnostic methods, and operative techniques per BMI category

\begin{tabular}{|c|c|c|}
\hline \multirow[t]{2}{*}{ BMI category } & \multicolumn{2}{|l|}{ Patient characteristics } \\
\hline & Mean age (SD) & Gender \\
\hline $\begin{array}{l}\text { Underweight } \\
n=36\end{array}$ & $11.0(4.07)^{*}$ & $\begin{array}{l}\text { Girls } 11(31 \%) \\
\text { Boys } 25(69 \%)\end{array}$ \\
\hline $\begin{array}{l}\text { Normal weight } \\
n=346\end{array}$ & $13.2(3.51)$ & $\begin{array}{l}\text { Girls } 162(47 \%) \\
\text { Boys } 184(53 \%)\end{array}$ \\
\hline $\begin{array}{l}\text { Overweight } \\
n=59\end{array}$ & $13.0(3.77)$ & $\begin{array}{l}\text { Girls } 29(49 \%) \\
\text { Boys } 30(51 \%)\end{array}$ \\
\hline $\begin{array}{l}\text { Obese } \\
n=16\end{array}$ & $12.4(3.61)$ & $\begin{array}{l}\text { Girls } 8(50 \%) \\
\text { Boys } 8(50 \%)\end{array}$ \\
\hline \multirow[t]{2}{*}{ BMI category } & \multicolumn{2}{|c|}{ Diagnostic methods and operative techniques } \\
\hline & Ultrasound & Operative techniques \\
\hline $\begin{array}{l}\text { Underweight } \\
n=36\end{array}$ & Performed $19(63 \%)^{* *}$ & $\begin{array}{l}\text { LA } 17(53 \%) \\
\text { OA } 15(47 \%)^{* * *}\end{array}$ \\
\hline $\begin{array}{l}\text { Normal weight } \\
n=346\end{array}$ & Performed $121(40 \%)$ & $\begin{array}{l}\text { LA } 226(73 \%) \\
\text { OA } 84(27 \%)\end{array}$ \\
\hline $\begin{array}{l}\text { Overweight } \\
n=59\end{array}$ & Performed $20(37 \%)$ & $\begin{array}{l}\text { LA } 39(72 \%) \\
\text { OA } 15(28 \%)\end{array}$ \\
\hline $\begin{array}{l}\text { Obese } \\
n=16\end{array}$ & Performed $6(50 \%)$ & $\begin{array}{l}\text { LA } 10(67 \%) \\
\text { OA } 5(33 \%)\end{array}$ \\
\hline
\end{tabular}

The descriptive analyses of patient characteristics, diagnostic methods, and operative techniques were all corrected for the year in which the patient had been diagnosed with appendicitis

$L A$ laparoscopic appendectomy, $O A$ open appendectomy

$* P=0.001$ in underweight patients in comparison to normal weight patients

$* * P=0.023$ in underweight patients in comparison to normal weight patients

$* * * P=0.005$ in underweight patients in comparison to normal weight patients

\section{Influence of BMI on diagnosing appendicitis}

The descriptive analysis showed that underweight children had a negative appendectomy more often than patients with normal weight ( 28 versus $18 \%$, respectively). Multivariable analysis confirmed that underweight children had three times higher risk of a negative appendectomy than normal weight patients $(P=0.008)$ (Table 2$)$. Furthermore, the multivariable analysis with extra correction for ultrasound use showed that both underweight and the use of ultrasound influenced the negative appendectomy rate $(P=0.008$ and $P=0.004$, respectively). Underweight, however, had a negative influence, whereas ultrasound influenced the negative appendectomy rate positively. We found that obese children had a negative appendectomy rate of $25 \%$, although this was not significant when multivariable analysis was performed $(P=0.72)$. Apart from BMI, older children had a higher chance of a negative appendectomy (OR 1.11, 95 \% CI 1.03-1.20, $P=0.005$ ). Similarly, girls had a higher chance of experiencing a negative appendectomy than boys (OR 2.38, 95\%CI 1.43-3.98, $P<0.001$ ).

Moreover, a perforated appendicitis was observed more often in underweight children $(31 \%)$ than in normal weight patients $(20 \%)$, although this difference was not significant 
Table 2 The influence of BMI on diagnosing appendicitis

\begin{tabular}{|c|c|c|c|c|}
\hline \multirow[t]{2}{*}{ BMI category } & \multicolumn{4}{|l|}{ Negative appendectomies } \\
\hline & Number of patients & OR & $95 \% \mathrm{CI}$ & $P$ \\
\hline Underweight & $10 / 36(28 \%)^{*}$ & 3.00 & $1.29-6.94$ & $0.008 *$ \\
\hline Normal weight & $61 / 346(18 \%)$ & Ref. & Ref. & - \\
\hline Overweight & $6 / 59(10 \%)$ & 0.504 & $0.203-1.25$ & 0.13 \\
\hline Obese & $4 / 16(25 \%)$ & 1.76 & $0.523-5.90$ & 0.72 \\
\hline \multirow[t]{2}{*}{ BMI category } & Perforations & & & \\
\hline & Number of patients & OR & $95 \% \mathrm{CI}$ & $P$ \\
\hline Underweight & $11 / 36(31 \%)$ & 1.62 & $0.748-3.52$ & 0.24 \\
\hline Normal weight & $70 / 346(20 \%)$ & Ref. & Ref. & - \\
\hline Overweight & $7 / 59(12 \%)$ & 0.523 & $0.227-1.20$ & 0.14 \\
\hline Obese & $3 / 16(18 \%)$ & 0.872 & $0.241-3.160$ & 0.87 \\
\hline \multirow[t]{2}{*}{ BMI category } & Consultations & & & \\
\hline & Number of patients & OR & $95 \% \mathrm{CI}$ & $P$ \\
\hline Underweight & 1 consultation $30 / 30(100 \%)$ & $<0.001$ & $0.00-0.00$ & 0.99 \\
\hline Normal weight & $\begin{array}{l}>1 \text { consultations } 0 / 30(0 \%) \\
1 \text { consultation } 267 / 306(87 \%)\end{array}$ & Ref. & Ref. & - \\
\hline Overweight & $\begin{array}{l}>1 \text { consultations } 39 / 306(13 \%) \\
1 \text { consultation } 48 / 54(89 \%)\end{array}$ & 0.855 & $0.343-2.13$ & 0.92 \\
\hline \multirow[t]{2}{*}{ Obese } & $\begin{array}{l}>1 \text { consultations } 6 / 54(11 \%) \\
1 \text { consultation } 10 / 12(83 \%)\end{array}$ & 1.41 & $0.295-6.68$ & 0.63 \\
\hline & $>1$ consultations $2 / 12(17 \%)$ & & & \\
\hline
\end{tabular}

The influence of BMI on diagnosing appendicitis measured in terms of the number of negative appendectomies, perforations, and consultations required to diagnose the patient. The multivariate regression analysis was corrected for year, age, and gender

Ref. the reference group, i.e., the group of patients with normal weights with which each one of the other three BMI categories was compared, OR odds ratio, $95 \% C I 95 \%$ confidence interval

$* P<0.05$
$(P=0.24)$ (Table 2$)$. Overweight and obesity also did not have a significant influence on perforation rate.

There was no correlation between BMI and the number of consultations required for diagnosing appendicitis (Table 2).

\section{Influence of BMI on treatment of appendicitis}

Underweight and obese patients had the highest complication rate after appendectomy, i.e., $25 \%$ in both cases (Table 3 ). The multivariable analyses, however, showed that the difference in complication rate was only significant for the underweight patients, since they had three times higher risk of complications compared to normal weight children $(P=0.041)$. Underweight patients experienced the following complications: wound infection $(6 \%)$, abscess $(8 \%)$, peritonitis (3\%), fever $(5 \%)$, bowel obstruction $(3 \%)$, urinary tract problem $(6 \%)$, postoperative ileus $(3 \%)$, readmission $(6 \%)$, and reoperation $(6 \%)$.

Univariate and multivariable analyses revealed that underweight and obese patients needed to stay in the hospital significantly longer than patients with normal weight: a median hospital stay of 4.5 and 3.5 days compared to 3.0 days $(P=0.001$ and $P<0.001)$, respectively (Table 3$)$.

\section{Influence of BMI on surgical outcome in correlation to operative techniques}

First, we studied whether there was a difference in surgical outcome after laparoscopic and open appendectomy in general, prior to subdividing the children into different BMI categories. We found that $17 \%$ of the children experienced complications after laparoscopic appendectomy and $13 \%$ after open appendectomy $(P=0.51)$. For both laparoscopic and open appendectomy, the median length of hospital stay was 3.0 days $(P=0.81)$.

Subsequently, we analyzed the influence of the operative technique among different BMI groups to determine the most suitable technique for each group. We observed that the median length of hospital stay and complication rate of underweight patients who had undergone either laparoscopic or open appendectomy was not significantly different $(P=0.79$ and $P=0.059$, respectively). Similarly, the length of hospital stay and complication rate were not significantly different for overweight patients ( $P=0.58$ and $P=0.65$, respectively).

We did, however, find a significant difference between the lengths of hospital stays in obese patients who had undergone either laparoscopic or open appendectomy $(P<0.001)$. After 
Table 3 The influence of BMI on the treatment of appendicitis

\begin{tabular}{lllll}
\hline BMI category & Complications & & & \\
& Number of patients & OR & $95 \%$ CI & $P$ \\
Underweight & $9 / 36(25 \%)$ & 2.75 & $1.03-7.35$ & $0.041^{*}$ \\
Normal weight & $52 / 346(15 \%)$ & Ref. & Ref. & - \\
Overweight & $5 / 59(9 \%)$ & 0.655 & $0.217-1.98$ & 0.44 \\
Obese & $4 / 16(25 \%)$ & 2.08 & $0.391-11.0$ & 0.39 \\
BMI category & Length of hospital stay & & & \\
& Median of days (min-max) & $B$ & $95 \%$ CI & $P$ \\
Underweight & 4.5 days (1-24) & 2.34 & $0.797-3.89$ & $0.001^{*}$ \\
Normal weight & 3.0 days (0-23) & Ref. & Ref. & - \\
Overweight & 2.0 days $(1-13)$ & -0.064 & $-1.21-1.08$ & 0.80 \\
Obese & 3.5 days (0-55) & 9.10 & $6.55-11.7$ & $<0.001^{* *}$ \\
\hline
\end{tabular}

The influence of BMI on the treatment of appendicitis as measured in terms of complication rate and length of hospital stay. The multivariate regression analysis was corrected for year, age, gender, operative technique, perforation status, CRP levels, and leukocyte levels

Ref. the reference group, i.e., the group of patients with normal weights with which each one of the other three BMI categories was compared, $O R$ odds ratio, $95 \% C I 95 \%$ confidence interval, $B$ regression coefficient $* P<0.05 ; * * P<0.001$

laparoscopic appendectomy, obese patients stayed in the hospital for a median of 2.5 days (interquartile range 1.75-16.75), compared to 3.0 days in patients with normal weights. Obese patients, who had undergone open appendectomy, stayed in the hospital for a median of 4.0 days (interquartile range 3.0$6.0)$, compared to patients with normal weights who stayed in the hospital for 3.0 days (Fig. 1). There was no difference in complication rate between obese and normal weight children when operative technique was taken into account $(P=0.99)$.

\section{Discussion}

We demonstrated that underweight significantly hampers the accuracy of diagnosing appendicitis by increasing the

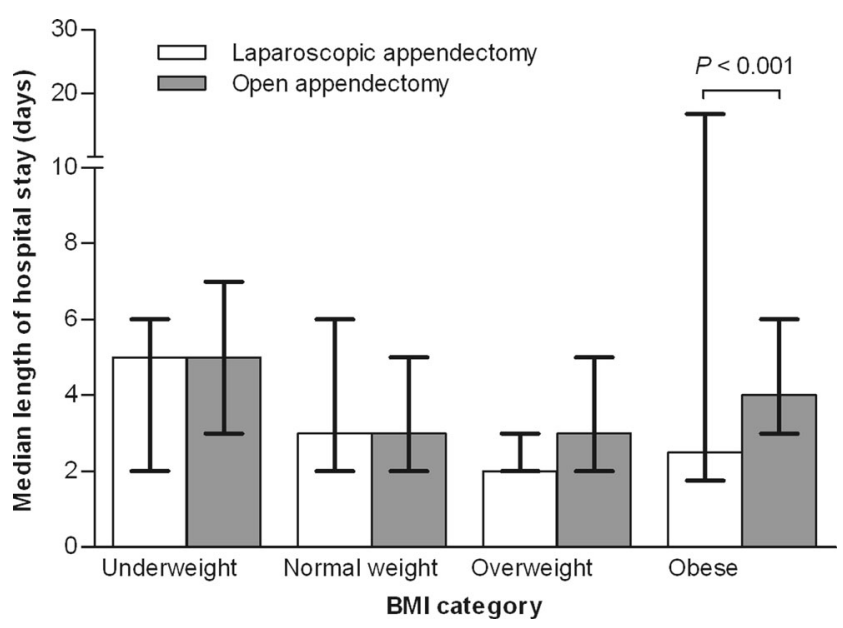

Fig. 1 Influence of BMI on the length of hospital stay after laparoscopic and open appendectomy. Length of hospital stay was measured in terms of median days with interquartile range negative appendectomy rate. Furthermore, both underweight and obesity seemed to negatively influence the treatment of appendicitis by increasing the complication rate and length of hospital stay. Finally, our data confirmed that laparoscopic appendectomy should be the treatment of choice for obese children, since it is associated with a shorter stay in hospital.

Multiple factors are known to influence negative appendectomy rates, for instance age and gender [19], as was confirmed by our study. Therefore, to properly investigate the correlation between BMI and negative appendectomy rate, we corrected our analysis for these factors. We found that underweight patients had a three times higher chance of a negative appendectomy than normal weight children. Other BMIs had no significant influence on the negative appendectomy rate. The negative appendectomy rate can also be influenced by the number and type of diagnostic tools used to diagnose appendicitis. In this study, patients from all four BMI groups were given a standard clinical and laboratory assessment. The underweight group, however, was examined more often by ultrasound than children from other BMI groups. Even when corrected for the possible influence of year of appendectomy, age, gender, and the use of ultrasound, we found that underweight children, independent of all those factors, still had a significantly higher risk of a negative appendectomy. It is important to note that underweight does not seem to influence the accuracy of ultrasound [20, 21]. We conclude, therefore, that even though underweight children were examined more extensively, they were nevertheless misdiagnosed with appendicitis more often than children from the other BMI groups. Although we cannot explain the aforementioned finding, we think it could be caused by the fact that children with underweight are more sensitive to abdominal examination. Consequently, the physician might think that underweight patients experience more 
pain during examination than normal weight and obese children. The alternative diagnoses for the children with negative appendectomies were not further investigated in this study.

Delay in an accurate diagnosis and the consequent delay of treatment results in perforation of the appendix. According to the literature, obesity may increase perforation rate [2]. We did not observe an increased perforation rate in any of the BMI groups. The discrepancy with another study [2] could possibly be explained by the differences in the statistical analyses used. We analyzed four different BMI groups, whereas Blanco et al., for instance, investigated two groups of patients: obese and non-obese [2]. Additionally, various hospitals use different methods to diagnose appendicitis, which may influence the time invested in accurate diagnosis and, therefore, perforation rate [22].

We found that the number of consultations required to diagnose appendicitis was not influenced by BMI. Our study is corroborated by a previous analysis where no significant difference between obese and non-obese patients was found in terms of consultations required to diagnose and perforation rate [17].

The potential influence of BMI on the complication rate after appendectomy is still a subject of debate. We found that in comparison to normal weight children, obese children experience a relatively large number of complications after appendectomy. This difference was, however, not significant, due probably to the small number of obese patients included in our study. We did find a significant difference in complication rate between underweight and normal weight children, i.e., underweight children had an almost three times higher risk of complications. Therefore, alongside obesity, one should also consider underweight as a risk factor for complications. Although interesting, we did not analyze in detail to which type of complications underweight children were prone, as the groups of patients with different complications were too small.

Quality of treatment can also be measured by considering the length of hospital stays. We found that hospital stays were significantly longer in both obese and underweight children, due possibly to their higher complication rates, although other factors might also have influenced the length of hospital stays. Staying for an additional 1-2 days in case of patients with underweight and obesity is associated with higher financial costs, which implies economic consequences.

Furthermore, we also found that obesity was associated with significantly prolonged length of hospital stays in patients who had undergone open appendectomy, rather than in case of laparoscopic appendectomy. We conclude, therefore, that laparoscopic appendectomy is more favorable for obese pediatric patients than open appendectomy, as has been reported for obese adults and children [6, 14, 15]. Mason et al. postulated that the advantage of the laparoscopic technique in obese patients is entirely due to wound and wound-related complications [23]. In line with this, since obese children often suffer from diabetes, the process of wound healing is worse than that in children with normal weight or underweight. It would be interesting to investigate in a follow-up study whether the occurrence of complications and length of hospital stays are increased in a subpopulation of obese children with diabetes.

\section{Final conclusion}

In this study, we demonstrate that underweight increases the negative appendectomy rate significantly and that it should be considered a risk factor for the misdiagnosis of appendicitis. Since both underweight and obese patients showed a tendency toward having increased complication rates and longer hospital stays, we conclude that both underweight and obesity negatively influence the outcome of appendectomy. Since we found that age as well as gender influenced the chance of having a negative appendectomy, we conclude that BMI is not a single factor affecting the diagnosis and treatment of appendicitis. Lastly, laparoscopic appendectomy is recommended in case of obese children in order to decrease the length of hospital stays.

Acknowledgments The authors thank $\mathrm{M}$. Trzpis, $\mathrm{PhD}$, for critically reviewing the manuscript and T. van Wulfften Palthe, $\mathrm{PhD}$, for editing and correcting the English used in the manuscript.

\section{Compliance with ethical standards}

Conflict of interest The authors declare that they have no conflict of interest.

Source of funding No external funding was secured for this study.

Financial disclosure None of the authors have financial relationships relevant to this article to disclose.

Ethical approval The local medical ethics committee of the University Medical Center Groningen approved this study.

All procedures performed in studies involving human participants were in accordance with the ethical standards of the institutional and/or national research committee and with the 1964 Helsinki Declaration and its later amendments or comparable ethical standards.

For this retrospective study, formal consent was not required.

Open Access This article is distributed under the terms of the Creative Commons Attribution 4.0 International License (http:// creativecommons.org/licenses/by/4.0/), which permits unrestricted use, distribution, and reproduction in any medium, provided you give appropriate credit to the original author(s) and the source, provide a link to the Creative Commons license, and indicate if changes were made. 


\section{References}

1. Kutasy B, Puri P (2013) Appendicitis in obese children. Pediatr Surg Int 29:537-544

2. Blanco F, Sandler A, Nadler E (2012) Increased incidence of perforated appendicitis in children with obesity. Clin Pediatr 51:928932

3. Kutasy B, Hunziker M, Laxamanadass G, Puri P (2010) Increased incidence of negative appendectomy in childhood obesity. Pediatr Surg Int 26(10):959-962

4. Falagas M, Kompoti M (2006) Obesity and infection. Lacet Infect Dis 6:438-446

5. Davies D, Yanchar N (2007) Appendicitis in the obese child. J Pediatr Surg 42:857-861

6. Mason R, Mason A (2013) Open-close case? New data on appendectomy in an obese patient cohort. Expert Rev Gastroenterol Hepatol 7:1-3

7. Setzer N, Saade E (2007) Childhood obesity and anesthetic morbidity. Paediatr Anaesth 17:321-326

8. Deugarte A, Stark R, Kaji A, Yaghoubian A, Tolan A, Lee S (2012) Obesity does not impact outcomes for appendicitis. Am Surg 78: 254-257

9. Hoffmann M, Lefering R, Gruber-Rathmann M, Rueger J, Lehmann W (2012) The impact of BMI on polytrauma outcome. Injury 43:184-188

10. Bialo S, Gordon C (2014) Underweight, overweight and pediatric bone fragility: impact and management. Curr Osteoporos Rep 12: 319-328

11. M. Rytter, L. Kolte, A. Briend, H. Friis, V. Christensen (2014) The immune system in children with malnutrition-a systematic review. PLoS One 25

12. Thompson C, Fuhrman M (2005) Nutrients and wound healing: still searching for the magic bullet. Nutr Clin Pract 20:331-347

13. J. Ho, A. Wu, M. Lee, S. Lau, P. Lam, S. Kwok, R. Kwan, C. Lam, C. Tam, S. Lee (2014) Malnutrition risk predicts surgical outcomes in patients undergoing gastrointestinal operations: results of a prospective study. Clin Nutr S0261-5614
14. Kutasy B, Hunziker M, Laxamanadass G, Puri P (2011) Laparoscopic appendectomy is associated with lower morbidity in extremely obese children. Pediatr Surg Int 27:533-536

15. H. Masoomi, N. Nguyen, M. Dolich, L. Wikholm, N. Naderi, S. Mills, M. Stamos (2011) Comparison oflaparoscopic versus open appendectomy for acute nonperforated and perforated appendicitis in the obese population. Am J Surg 202: 733-8-discussion 738-9

16. Clarke T, Katkhouda N, Mason R, Cheng B, Olasky J, Sohn H, Moazzez A, Algra J, Chaghouri E, Berne T (2011) Laparoscopic versus open appendectomy for the obese patient: a subset analysis from a prospective, randomized, double-blind study. Surg Endosc 25:1276-1280

17. Towfigh S, Chen F, Katkhouda N, Kelso R, Sohn H, Berne T, Mason R (2008) Obesity should not influence the management of appendicitis. Surg Endosc 22:2601-2605

18. CBO (2008c) Kwaliteitsinstituut voor de Gezondheidszorg. Richtlijn Diagnostiek en behandeling van obesitas bij volwassenen en kinderen. 2015 (2008)

19. Bachur RG, Hennelly K, Callahan MJ, Chen C, Monuteaux MC (2012) Diagnostic imaging and negative appendectomy rates in children: effects of age and gender. Pediatrics 129(5):877-884

20. Abo A, Shannon M, Taylor G, Bachur R (2011) The influence of body mass index on the accuracy of ultrasound and computed tomography in diagnosing appendicitis in children. Pediatr Emerg Care 27(8):731-736

21. Yiğiter M, Kantarci M, Yalçin O, Yalçin A, Salman AB (2011) Does obesity limit the sonographic diagnosis of appendicitis in children? J Clin Ultrasound 39(4):187-190

22. Cobben L, Bakker O, Puylaert J, Kingma L, Blickman J (2009) Imaging of patients with clinically suspected appendicitis in the Netherlands: conclusions of a survey. $\mathrm{Br} \mathrm{J}$ Radiol 82:482-485

23. R. Mason, A. Moazzez, J. Moroney, N. Katkhouda (2012) Laparoscopic vs open appendectomy in obese patients: outcomes using the American College of Surgeons National Surgical Quality Improvement Program database. J Am Coll Surg 215: 88-99-discussion 99-100 